\title{
Diversity Slam
}

\section{November 2013, Berlin}

Am 28./29. November 2013 fand in Berlin rund um das Thema Vielfalt eine Diversity-Konferenz statt, die der Tagesspiegel gemeinsam mit der Charta der Vielfalt zum zweiten Mal ausrichtete. Es diskutierten rund 300 Experten über Vielfalt in deutschen Unternehmen und Institutionen. Erstmalig gab es einen Diversity Slam, dessen Ziel es war, Wissen zur Bühnenshow zu machen. Mehr als 30 Projekte und Initiativen hatten sich mit Beiträgen über Geschlecht, Nationalität, ethnische Herkunft, Religion, Behinderung, Alter und sexuelle Orientierung beworben. Vertreter/innen der Deutschen Bahn, der Charta der Vielfalt und des Tagesspiegels wählten vier Beiträge für das Finale am 28. November 2013 in Berlin aus. Dabei waren PrOut@ Work, Success Across, der Deutsche Juristinnenbund e.V. (djb) und die Siegerin des Wettbewerbs Uta Zech mit ihrem Thema „Älterwerden in Unternehmen“.

Jedem antretenden Projekt standen zehn Minuten zur Verfügung, um seine Initiative zum Thema Vielfalt in Unternehmen, Organisationen und Gesellschaft kurzweilig, unterhaltsam und leicht verständlich vorzustellen - „, alle Hilfsmittel erlaubt“. Das Publikum bildete die Jury, die mit Wertungen von eins bis zehn über den ersten Platz entschied. Es wurde ein knappes, umso furioseres Rennen:

Für den djb trat Manuela Schauerhammer auf. Sie präsentierte das Projekt „Aktionärinnen fordern Gleichberechtigung “ in einer das Publikum unmittelbar einbindenden „szenischen Lesung “: Hierfür stellte sie eine Aktionärsversammlung mit
Original-Hauptversammlungs-Zitaten nach, die die Diskriminierung von Frauen in Unternehmen nur allzu deutlich machten. Das Publikum war von Manuela Schauerhammer eingangs aufgefordert worden, sich direkt zu beteiligen und die Szenerie unmittelbar zu kommentieren: Zu johlen, zu klatschen oder zu buhen. Das Konzept ging auf: Spätestens, als Manuela Schauerhammer mit Verve Erdnüsse in die Menge warf - „Peanuts“, die ja bekanntlich von DAX-Vorstandsvorsitzenden mindestens im übertragenen Sinne allzu geschätzt werden - waren die Zuschauenden in den Bann des djb-Projekts gerissen. Die Begeisterung für den Beitrag, aber auch das echte Interesse am Thema zeigte sich schließlich nicht nur in der Bewertung - nach Punkten lag der djb am Ende nur äußerst knapp hinter der Erstplatzierten sondern vor allem auch an den vielen Anschlussgespräche sowie daran, dass sämtliche Informationsbroschüren und Flyer des djb zum Projekt „Aktionärinnen fordern Gleichberechtigung“ in wenigen Minuten ausgegeben waren.

„Wir fanden es spannend, ein so ernstes Thema in einem modernen, etwas anderem Licht präsentieren zu können “, sagte Manuela Schauerhammer. „Uns ging und geht es darum, eine Sensibilisierung und eine noch größere Öffentlichkeit für das Thema Gleichstellung zu erreichen. “ Nicht mit trockenen Zahlen, sondern „mit einem Schmunzeln“ - Humor brauche man schließlich auch, um mit den zum Teil unfassbaren Antworten der Unternehmensvertreter auf den echten Hauptversammlungen umzugehen. (AG)

\section{„Stolz und Vorurteil“}

\section{Anmerkungen zum Diskurs über „Frauen in Führungspositionen“}

\section{Katrin Lange}

Mitarbeiterin, Deutscher Juristinnenbund e.V., Berlin

„[N]iemand ist den Frauen gegenüber arroganter, aggressiver oder verächtlicher als ein in seiner Männlichkeit verunsicherter Mann. Die Männer, die sich von ibresgleichen nicht eingeschüchtert fühlen, sind auch viel eher bereit, die Frau als ihresgleich anzuerkennen."1

Simone de Beauvoir, 1949

Viel ist in den vergangenen Jahren über die Einführung einer gesetzlichen Frauenquote als adäquates Mittel zur Erhöhung des Anteils von Frauen in Führungspositionen gestritten worden. Jetzt, wo ein Gesetz auf Grund veränderter Mehrheitsverhältnisse im Bundestag auf den Weg gebracht wird ${ }^{2}$, beschäftigt sich dieser Artikel mit medial verbreiteten Positionen, die keinen zwingenden Änderungsbedarf in unserer Gesellschaft erkennen wollen. Im Gegenteil: Es werden Argumente angeführt, die den gegenwärtig als „,ausreichend“ be- fundenen Zustand anhaltend verteidigen bzw. die mitunter auch einen unvermeidbaren Geschlechterkonflikt heraufbeschwören, sollte sich an diesem Zustand zugunsten der Frauen etwas ändern.

Fakt ist, wenn es darum geht, in Deutschland Verantwortung zu tragen, Macht auszuüben, Einfluss zu nehmen und oder Geld zu verdienen, haben Frauen von vornherein geringere Chancen als Männer. Die Strukturen und Mechanismen dieses zementierten Verhältnisses wurden in zahlreichen Studien aufgedeckt und werden fortwährend auch immer wieder neu belegt. ${ }^{3}$

1 Beauvoir, Simone de: Das andere Geschlecht. Sitte und Sexus der Frau, Rowohlt Verlag, 1998, S. 22.

2 Am 25. März 2014 stellten Bundesfamilienministerin Manuela Schweswig und Bundesjustizminister Heiko Maas gemeinsam die Leitlinien für das Gesetzgebungsverfahren „Gesetz für die gleichberechtigte Teilhabe von Frauen und Männern an Führungspositionen in der Privatwirtschaft und im Öffentlichen Dienst“" in Berlin vor. Das Gesetz soll 2015 in Kraft treten.

3 S. u.a. folgende Publikationsliste: http://www.djb.de/Projekt_HV/ Publikationen/Studien/ (Zugriff: 14.3.2014). 
Verschiedene Akteure, allen voran zivilgesellschaftliche und wissenschaftliche Organisationen, arbeiten in stetigen Schritten an der Herstellung von Gleichberechtigung in dieser Gesellschaft. Und keine Angst, wir sind noch nicht fertig; auch wenn eine lang formulierte Forderung nach der Einführung einer gesetzlichen Frauenquote nun endlich Gehör in der Politik gefunden hat!

Parallel hierzu hat sich ein weiterer Strang manifestiert, der den Erkenntnissen und Erfahrungen über ungleich verteilte Karrierechancen von Frauen und Männern in Deutschland diametral entgegensteht. Auffällig häufig zirkulieren dabei dem Anschein nach rationale und belastbare Argumente, wenn es um die Herstellung gleichberechtigter Teilhabe von Frauen an der Gesellschaft geht: Allen voran das beliebte Standardargument, es mangele schlichtweg an qualifizierten Frauen, um Führungspositionen mit ihnen zu besetzen. Aber auch gern genommene Erklärungen, dass Frauen anders seien, weniger Präsenz im Job zeigen ${ }^{4}$ bzw., wenn wir schon einmal dabei sind, eigentlich doch auch gar keine Karriere machen wollen ${ }^{5}$. Argumentationen dieser Art zeigen überdeutlich, dass die berufliche Diskriminierung von Frauen tief verwurzelt ist. So tief, dass diese an der Oberfläche oftmals nur als ein von den Frauen selbst verschuldetes Dilemma erscheint.

Teilweise wird jedoch auch der Boden der angeblich so vorherrschenden „Realität“ verlassen und regelrechte Angstszenarios entworfen: So wird die öffentlichkeitswirksame Beförderung von wenigen Frauen in die Topetagen von Unternehmen zum Anlass genommen von einer „Welle der Weiblichkeit“ ${ }^{* 6}$ zu sprechen. In einer regelrechten „Besetzungshysterie“ sorgen heutige Personalentscheidungen für ,gefällige Abkürzungen für Frauen“.7 Oder, umgekehrt, eine ganze Generation der heute zwischen 30 - und 45-Jährigen Männer wird per se verloren gegeben. ${ }^{8}$ Männer gehen in Scharen „über Board“9 oder sie „schlagen zurück“10, weil sie die „Schnauze voll haben von der Gleichberechtigung “11. Massenpanik herrscht dann spätestens, wenn die „Quoten-Frauen“ kommen, und dass leider nicht nur bei den Männern. ${ }^{12}$

Im Kern zielen alle diese lautstark verkündeten Parolen darauf ab, aus der fortschreitenden Emanzipation der Frauen ein Nullsummenspiel für die Männer zu generieren: Die besondere Berücksichtigung einer bestimmten Gruppe ergebe notwendigerweise eine Beeinträchtigung der Chancen Anderer. Dieser Gedanke, übertragen auf das „Schlachtfeld“ der Wirtschaft, wo sich seit wenigen Jahren die Unternehmen dem Thema „Frauen in Führungspositionen“ - zumindest verbal angenommen haben - impliziert nichts anderes als einen heraufbeschworenen Geschlechterkonflikt ${ }^{13}$ um Aufstiegschancen. In den sowieso schon bestehenden „männlichen Verteilungskampf“ um die wenigen Führungspositionen verschärfe sich der Wettbewerb, wenn Frauen dabei jetzt auch noch mitmischen wollen. Oder, um mit den Worten von djb-Präsidentin Ramona Pisal zu sprechen: „Es geht um Macht, Einfluss und Geld. Freiwillig wird nicht geteilt.“

Die Männer derweil geraten über die ihnen zukünftig in Aussicht gestellten, „geringeren“ Karrierechancen regelrecht in eine „Gratifikationskrise“. Demnach erkrankt diejenige Person, die trotz außerordentlichem Einsatz für eine Sache, keine angemessene Belohnung erhält. ${ }^{14}$ Im Umkehrschluss würde dies bedeuten, dass sämtliche Frauen, nicht nur an „Gläserne
Decken und oder Wände“ stoßen, sondern darüber auch noch ihre Gesundheit einbüßen. Also schnell weg von der Pathologisierung eines strukturellen Problems der hiesigen Arbeitswelt. ${ }^{15}$

4 „Frauen wird von Personalverantwortlichen eine stärkere Verantwortung für die Familien und damit geringere Verfügbarkeit für die Anforderungen der Führungsposition und eine Karriereunterbrechung auf Grund von Familienaufgaben unterstellt, obwohl dies längst empirisch widerlegt ist (Müller, Ursula, 1999: Zwischen Licht und Grauzone: Frauen in Führungspositionen, in: Arbeit, 8. Jg., Heft 2, S. 137-161-Anm. d. Verf.) und etwa die Hälfte der angestellten Managerinnen überhaupt keine Kinder hat (Bischoff, Sonja, 2005: Wer führt in (die) Zukunft? - Anm. d. Verf.)", in: von Alemann, Annette: Chancenungleichheit im Management, in: Sozialwissenschaften und Berufspraxis (SuB), 30. Jg. (2007), Heft 1, S. 26.

5 Dieses Argument wird oftmals nur verkürzt dargestellt. Etwaige Äußerungen von Frauen beziehen auch die vorherrschenden Bedingungen mit ein, unten denen heutzutage eine Karriere zu bewerkstelligen ist.

6 Vgl. Werle, Klaus: Mann über Board, in: manager magazin v. 26.10 .2012

7 Ebd.

8 Vgl. Weiguny, Bettina: Lauter verlorene Männer, in: FAZ v. 2.6.2012.

9 Vgl. Werle, Klaus: Mann über Board, in: manager magazin v. 26.10.2012.

10 Führungspositionen nur für Frauen? Die Männer schlagen zurück, in: Brigitte 03/2014 v. 10.1.2014.

11 Studie: Männer haben von Gleichberechtigung die Schnauze voll, in: Focus v. 30.9.2013.

12 Vgl. Wolff, Reinhard: Frauenquotengegnerin Annika Falkengren. Europäische Topbankerin, in: taz v. 19.11.2013; Henkel: Chefin Simone Bagel-Trah gegen Frauenquote aber für Mittel gegen Haarausfall, in: netz trends v. 8.12.2013; Eon-Personalchefin lehnt Frauenquote ab, in: Handelsblatt v. 31.5.2013; Siemens-Personalchefin lehnt Frauenquote $a b$, in: Focus v. 17.10.2011, Klonovsky, Michael: Bitte keine Quoten-Frauen!, in: Focus v. 27.06.2010. Nicht nur Männer, sondern auch viele Frauen positionieren sich öffentlich gegen die Quote. Auf Grund der negativen Konnotation die das Wort „Quotenfrau“ im medialen Diskurs erhalten hat, könnte einer der Gründe für diese Positionierung darin bestehen, die eigene Qualifikation und Leistung vor Diskreditierung zu bewahren. Wohingegen die Positionen von Wirtschaftsvertreterinnen zur Quote vielmehr deren Unternehmenspolitik (und somit auch die bestehende Unternehmenskultur) wiedergeben. Inwiefern sich darin auch eine persönliche Einstellung zur Quote widerspiegelt, lässt sich kaum Ermessen.

13 Knauß, Ferdinand: Geschlechterkonflikt beim Daimler, in: Wirtschaftswoche v. 5.4.2013; Werle, Klaus: Mann über Board, in: manager magazin v. 26.10.2012; Schmergal, Cornelia/Tödtmann, Claudia/Wildhagen, Andreas: Geschlechterkampf: Die Frauenquote bremst Männer aus, in: Wirtschaftswoche v. 14.8.2012.

14 „Nach dem Modell der Gratifikationskrise erkrankt eine Person dann, wenn sie sich stark verausgabt und dafür nicht in angemessener Weise entschädigt wird. Wenn der eigene Einsatz (etwa in Form von Engagement, Wissen, Zeit, Identifikation, Leistung und Persönlichkeit) nicht durch entsprechende Belohnung (etwa in Form von ausbildungsadäquater Beschäftigung, Lohngerechtigkeit, Arbeitsplatzsicherheit, Weiterbildungs-, Karriere- und Einflussmöglichkeiten) kompensiert wird, so entstehe dadurch das als „Gratifikationskrise“ bezeichnete Krankheitspotential“, in: Wikipedia, Zugriff: 21.3.2014.

15 Inwiefern die Einführung einer Frauenquote Einfluss auf Männer hat, damit beschäftigt sich aus medizinisch-psychologischer Sicht der Verband der Betriebs- und Werksärzte (VDBW). In dem Artikel „Lauter verlorene Männer" von Bettina Weiguny (FAZ v. 2.6.2012) prophezeit Dr. med. Anette Wahl-Wachendorf, VDBWVizepräsidentin, das „Resignation, Angst und Zynismus der Männer am Arbeitsplatz ein Riesenthema werde". Auf Nachfrage des djb erklärt sie den Hintergrund des Zitates aus den Erfahrungen der betriebsärztlichen Kollegenschaft in der Sprechstunde, d.h. Äußerungen von männlichen Beschäftigten. Eine repräsentative Studie des Verbands liegt jedoch noch nicht vor. 
„[M]an kann es ibnen [den Männern - Anm. d. Verf.] nicht verübeln, dass sie all die Annehmlichkeiten, [...], nicht leichten Herzens opfern; sie wissen, was sie verlieren, [...]; sie wissen aber nicht, was die Frau von morgen ihnen bringen wird. " 16

Simone de Beauvoir, 1949

Dass die Frauenanteile auf den einzelnen Führungsebenen, in den Vorständen und Aufsichtsräten der Unternehmen konstant auf einem niedrigem Niveau verharren, hat der Deutsche Juristinnenbund e.V. (djb) mit Veröffentlichung seiner Abschlusspublikation zum Projekt „Aktionärinnen fordern Gleichberechtigung“ jüngst erneut nachgewiesen. Mehr noch, die Auswertung von 75 börsennotierten Unternehmen in Deutschland, darunter alle 30 DAX-Unternehmen, deren Hauptversammlungen in den vergangenen vier Jahren jährlich besucht wurden, ergibt, dass nicht nur die Veränderungen in den Unternehmen zu gering sind, sondern vor allem die Wirksamkeit bestehender Instrumente zur Erhöhung des Frauenanteils in Führungspo-

\section{Ergo, ob befürwortet oder abgelehnt, die Unternehmen werden gezwungen sein, sich in Zukunft ernsthaft mit der Herstellung gleichberechtigter Karrierechancen von Frauen und Männern auseinanderzusetzen.}

sitionen auf allen Managementebenen angezweifelt werden muss. ${ }^{17}$ Im Übrigen auch ein Argument gegen die Vielzahl bereits bestehender Maßnahmen zur „Frauenförderung“ in den Unternehmen: Zum einen führen sie offensichtlich nicht zu den damit verbundenen Zielen. Zum anderen geben sie dem Vorurteil Aufwind, dass Frauen Defizite haben, die mit speziellen Coaching-, Mentoring- und Seminarangebote beseitigt werden müssten. Eine signifikante Verbesserung kann nach Einschätzung des djb demnach nur durch eine gesetzliche Regelung erfolgen. Nun soll im Folgenden nicht das Für und Wider einer Frauenquote diskutiert werden. Ein entscheidender Schritt auf der politischen Ebene ist jüngst angestoßen worden. Ergo, ob befürwortet oder abgelehnt, die Unternehmen werden gezwungen sein, sich in Zukunft ernsthaft mit der Herstellung gleichberechtigter Karrierechancen von Frauen und Männern auseinanderzusetzen.
Weg von öffentlichkeitswirksam formulierten Lippenbekenntnissen der obersten Managementriege, trägt diese Entwicklung - sofern sie zu einem akzeptierten und festen Bestandteil einer jeden Unternehmenskultur wird - das Potential, die Mechanismen struktureller Diskriminierung von Frauen im Erwerbsleben offenzulegen und sich für deren Aufhebung gemeinsam zu engagieren. Die Quote könnte in diesem Zusammenhang nicht als verhasstes „Männerverdrängungsinstrument“ - zu dem sie stets in verkürzten Debatten verteufelt wird fungieren, sondern als „Eisbrecher für verkrustete Arbeitsstrukturen “18. Aus dieser Perspektive ist die Quote nicht nur eine berufliche Chance für Frauen, um in Topmanagementpositionen aufzusteigen, sondern letztlich auch eine gesellschaftliche Chance, um grundlegende Strukturen der heutigen Arbeitsorganisation und -kultur in dieser Gesellschaft zum Wohle aller zu verändern.

Allem voran betrifft dies eine gleichmäßigere Aufteilung von Erwerbsarbeit und die Herbeiführung des Zweiverdienermodells. Ansätze hierfür wurden bereits u.a. vom Wissenschaftszentrum Berlin für Sozialforschung sowie vom Deutschen Institut für Wirtschaftsforschung in Berlin vorgelegt. ${ }^{19}$ Die Kernidee besteht darin, die heute noch vorherrschende Vollerwerbstätigkeit des Mannes zu reduzieren und als Ausgleich hierfür die überwiegende Teilzeiterwerbstätigkeit der Frau zu erhöhen, so dass die Wochenarbeitszeit beider Geschlechter 32 Stunden entspricht. Diese Neuaufteilung von Arbeitszeit führt den Expertinnen und Experten zu Folge zu einer höheren Lebenszufriedenheit. Darüber hinaus können Anschlussprobleme, wie Altersarmut von Frauen oder die überwiegend weibliche Beschäftigung im Niedriglohnsektor ange-

16 Beauvoir, Simone de: Das andere Geschlecht. Sitte und Sexus der Frau, Rowohlt Verlag, 1998, S. 22.

17 Aktionärinnen fordern Gleichberechtigung 2009 bis 2013. Mehr Frauen in Führungspositionen. Fazit und Forderungen, Berlin 2013. Weiteres s. Auszug des Kapitels „Fazit und Forderungen auf S. 49 in diesem Heft.

18 Rosenfeld, Dagmar: Frauenquote - Eisbrecher für verkrustete Strukturen, in: Rheinische Post v. 7.10.2013.

19 Baumgarten, Silke/Schütte, Sinja: 32 Stunden sind genug - Interview mit der Soziologin Jutta Allmendinger, in: Brigitte v. 7.12.2012. Müller, Kai-Uwe/Neumann, Michael/Wrohlich, Katharina: Bessere Vereinbarkeit von Familie und Beruf durch eine neue Lohnersatzleistung bei Familienarbeitszeit, in: DIW-Wochenbericht Nr. 46, 2013. 
gangen werden. Männern hingegen würde der Druck genommen, aus dem bis heute vorherrschenden Einernährermodell auszusteigen.

Aber die Quote kann noch weitere Effekte herbeiführen: Matthias Sutter, Professor für experimentelle Wirtschaftsforschung an der Universität Innsbruck, fand in einem von ihm durchgeführten Experiment heraus, dass Quotenregelungen das Verhalten von Frauen in Konkurrenzsituationen nachweislich positiv beeinflussen, ohne dabei das männliche Verhalten zu ändern. ${ }^{20}$ In dem Experiment sollten Frauen und Männer, gemischt verteilt in je einer Gruppe von sechs Probanden, innerhalb einer bestimmten Zeit Rechenaufgaben lösen. Im Anschluss folgten zwei Belohnungsmodelle: Das erste Modell richtete sich personenunabhängig nach der Anzahl der richtig gelösten Aufgaben. Das zweite Modell verdreifachte den Gewinn pro richtig gelöste Aufgabe, wenn die Person Gesamtsieger der Gruppe ist. Alle anderen erhielten, unabhängig von richtig gelösten Aufgaben, keine Belohnung. Das Modell ist also personenabhängig. In einem Artikel der Neuen Zürcher Zeitung erklärt Sutter das Ergebnis wie folgt: „In der normalen Situation ohne Quotenregelung, in der die zwei Sieger dadurch bestimmt wurden, wer in der Sechsergruppe die meisten Aufgaben richtig gelöst hatte, wählten 60 Prozent der Männer, aber nur 30 Prozent der Frauen die kompetitive Auszahlung. Das ist umso bemerkenswerter, weil es keinen objektiven Unterschied in den tatsächlichen Leistungen von Männern und Frauen gab. Im Fall einer Quotenregelung jedoch - entsprechend der die beste Frau auf jeden Fall einer der beiden Sieger war - wählten immer noch 60 Prozent der Männer die kompetitive Auszahlung, aber auch bereits 52 Prozent der Frauen. Eine Quotenregelung hatte also keinen Einfluss auf die Wettbewerbsbereitschaft von Männern, allerdings einen enorm starken auf die leistungsfähigsten Frauen. Während ohne Quotenregelung nur etwa 25 Prozent der bestqualifizierten Frauen den Wettbewerb wählten, waren es mit der Quotenregelung 80 Prozent. “21 Wichtig in diesem Zusammenhang ist die Ankündigung der Quotenregelung vor Eintritt der Wettbewerbssituation. Laut Sutter führe dies zu einem geschlechtsspezifischeren Wettbewerb, da sich die bestqualifizierten Frauen der Konkurrenzsituation zu Männern stellen, was sie im Falle einer fehlenden Regelung nicht getan hatten.

Daran anschließend fand der schwedische Ökonom Torsten Persson heraus, dass von einer Quotenregelung ${ }^{22}$ nicht nur die Frauen, sondern auch die Männer profitieren: „The academic and popular debate sometimes sees the goals of diversity and competence as in tension with one another. For example, in the debate on gender quotas, it is often claimed that a supply constraint for women results in a quota replacing competent men by mediocre women. We have argued, to the contrary, that achieving gender parity through quotas can actually promote competence by reducing the number of mediocre men." ${ }^{23}$ Die Quote wird oftmals und gern dahingehend missverstanden, dass durch sie inkompetente Frauen gegenüber den für die Führungspositionen qualifizierten Männern bevorzugt werden würden. Der djb hat stets darauf aufmerksam gemacht, gerade auch auf den von ihm besuchten Hauptversammlungen, dass dies mitnichten der Fall ist. In der Einführungsrede des djbFragenkatalogs aus dem Jahr 2012 heißt es: „Weil es immer wieder unseriös miteinander verknüpft wird, nur zur Klarstellung: Die Forderung nach einer Quote bedeutet nur, dass unter gleich qualifizierten Bewerbungen die Frau auszuwählen ist, wenn Frauen auf der fraglichen Führungsebene unterrepräsentiert sind. " 24 Persson geht in seiner Untersuchung weiter und bestätigte nicht nur einen vermuteten positiven Effekt einer Quote für Frauen, sondern eben auch für Männer. Aber nur, und das ist das Interessante an dieser Studie, wenn diese auch entsprechend qualifiziert für diese Position waren. ${ }^{25}$

Beide Forschungsergebnisse entkräften nicht nur die Mär von den unqualifizierten „Quotenfrauen“, sondern machen die Quote gesellschaftsfähig, da beide Geschlechter aufgrund ihrer Qualifizierung von einer Einführung dieser profitieren würden. In der Folge müssten sich die Unternehmen weiterhin an ihr gern und häufig propagiertes Credo halten, nicht Geschlecht, sondern Qualifikation und Leistung seien vorrangige Entscheidungsmerkmale für die Besetzung von Führungspositionen $^{26}$ - und dass, ohne wie bisher mit einer solchen Aussage intendiert, eine Quotenregelung für das Unternehmen damit von vornherein auszuschließen.

20 Balafoutas, Loukas/Sutter, Matthias: Affirmative Action Policies Promote Women and Do Not Harm Efficiency in the Laboratory, in Science, Volume 335, 3. Februar 2012, S. 579-582.

21 Sutter, Matthias: Quoten können das Verhalten der Frauen ändern, in: Neue Zürcher Zeitung v. 21.10.2012.

22 Die sozialdemokratische Partei Schwedens führte bei der Erstellung von Wahllisten für die Kommunalwahlen eine „Reisverschlussquote" ein bei der Frauen und Männer abwechselnd aufgeführt wurden.

23 Besley, Timothy/Folke, Olle/Persson, Torsten/Rickne, Johanna: Gender Quotas and the Crisis of the Mediocre Man: Theory and Evidence from Sweden, Universität Stockholm, 27.11.2012.

24 http://www.djb.de/Projekt_HV/Dokumentation/2012/ Fragenkatalog_2012/ (Zugriff: 31.3.2014).

25 „Kompetenz“ definieren die Wissenschaftler anhand verschiedener Kriterien, wie Ausbildung, Gehalt, politischer Erfolg bei Wahlen, innehabende Ämter etc.

26 Wie die Auswertungen der Protokolle von den Hauptversammlungen durchweg zeigen, gehört es zum Standardantwortrepertoire eines jeden Unternehmens auf diesen Glaubenssatz hinzuweisen, wenn es um das Thema „Frauen in Führungspositionen" geht. Vgl. auch Lange, Katrin: Aktionärinnen fordern Gleichberechtigung: Das Dogma von Qualifikation und Leistung - Einblicke in das djb-Projekt, in: djbZ 3/2012, S. 121-122. 